\title{
Structure, Composition Et Diversité Floristiques De La Forêt De La Djoumouna (République du Congo)
}

\section{Edmond Sylvestre Miabangana, PhD}

Herbier National du Congo (IEC), Institut National en Recherches Exactes et Naturelles (IRSEN), Cité scientifique de Brazzaville, République du Congo Agence Nationale de Valorisation des Résultats de la Recherche (ANVAR), Cité scientifique de Brazzaville, République du Congo

Doi:10.19044/esj.2020.v16n12p179 URL:http://dx.doi.org/10.19044/esj.2020.v16n12p179

\section{Résumé}

Une analyse floristique et structurale de la forêt de la Djoumouna ; dans la ceinture suburbaine, au sud-est de Brazzaville ; a été entreprise sur une superficie forestière de 10 hectares environ. L'objectif était d'approfondir la connaissance de la diversité floristique, par la caractérisation de la structure du peuplement forestier. 25 relevés de végétation ont été exécutés, en tenant compte des mesures dendrométriques de tous les individus ligneux de $\mathrm{DBH} \geq$ $10 \mathrm{~cm}$ à 1,30 $\mathrm{m}$ de hauteur du sol. Après traitement des données structurales, le peuplement forestier recèle une surface basale de $28 \mathrm{~m}^{2} /$ ha sur une densité moyenne de 653 individus provenant, de 123 espèces végétales et dont la famille des Leguminosae est la plus importante. Il réalise respectivement de 6,94 bits et 1,4 comme indices de diversité de Shannon-Weaver et d'équitabilité de Piélou. Deux espèces dominent l'ossature structurale, Pentaclethra eetveldeana De Wild. et T.Durand et Pentaclethra macrophylla Benth., qui réalisent les valeurs les plus élevées de surface terrière et d'Indice de Valeur d'Importance. Ces dernières réalisent respectivement 11 et 05 $\mathrm{m}^{2} /$ ha, et 54 et 30,5 de valeurs correspondantes. Ce trait justifie la dénomination structurale de cet îlot forestier. Ce peuplement forestier riche et diversifié, projette une bonne structuration, témoignant ainsi sa maturité. Ce trait milite en faveur de l'élaboration d'un plan d'aménagement à titre conservatoire.

Mots-clés : Richesse Et Diversité Floristiques, Végétation, Aménagement Forestier, Djoumouna 


\title{
Floristic Structure, Composition, and Diversity of the Djoumouna Forest (Republic of Congo)
}

\begin{abstract}
Edmond Sylvestre Miabangana, PhD
Herbier National du Congo (IEC), Institut National en Recherches Exactes et Naturelles (IRSEN), Cité scientifique de Brazzaville, République du Congo Agence Nationale de Valorisation des Résultats de la Recherche (ANVAR),

Cité scientifique de Brazzaville, République du Congo
\end{abstract}

\begin{abstract}
A floristic and structural analysis of the Djoumouna forest in the suburban belt, southeast of Brazzaville, was carried out in a forest area of about 10 hectares. This paper focuses on deepening the knowledge of plant diversity by characterizing the structure of the forest stand. 25 vegetation surveys were carried out, taking into account dendrometric measurements of all DBH woody individuals $\geq 10 \mathrm{~cm}$ at $1.30 \mathrm{~m}$ height from the ground. After processing the structural data, the forest stand has a basal area of $28 \mathrm{~m}^{2} / \mathrm{ha}$. This is with an average density of 653 individuals from 123 plant species, of which the Leguminosae family is the most important. It realizes 6.94 bits and 1.4 respectively as indices of diversity of Shannon-Weaver and evenness of Piélou. Two species dominate the structural framework, Pentaclethra eetveldeana (De Wild. and T.Durand) and Pentaclethra macrophylla (Benth). This achieves the highest basal area and Value Index of Importance values. The two species realize $11 \mathrm{~m}^{2} / \mathrm{ha}$ and $05 \mathrm{~m}^{2} / \mathrm{ha}$ respectively with corresponding values of 54 and 30.5. This feature justifies the structural denomination of the forest block. As a result of the rich and diversified forest population, a good structure is projected, thus testifying to its maturity. The feature militates in favour of the elaboration of a development plan as a precautionary measure.
\end{abstract}

Keywords: Plant Richness And Diversity, Vegetation, Forest Management, Djoumouna

\section{Introduction}

La dégradation des portions d'écosystèmes forestiers, urbains et périurbains, demeure l'une des préoccupations environnementales auxquelles sont confrontés les pays d'Afrique centrale (Marien, 2008). En ce qui concerne l'hinterland brazzavillois, sous l'effet conjugué de la croissance spatiale et 
démographique, les forêts matures ont été écrémées pour l'occupation et l'utilisation des sols. Ces dernières ont été supplantées par une végétation de substitution, formée entre autres, des vastes étendues de formations herbacées à Hyparrhenia diplandra (Hack.) Stapf, des jachères à Panicum maximum Jacq. et des formations synanthropiques à tendance nitrophile. Quant aux habitats ligneux de substitution, ce sont les fourrés à Sclerocroton cornutus (Pax) Kruijt et Roebers et Oncoba welwitschii Oliv. qui sont décelés sur terre ferme et ceux d'Alchornea cordifolia (Schumach. et Thonn.) Müll.Arg. et Trachyphrynium braunianum (K.Schum.) Baker, sur substrat édaphique de sol hydromorphe (Miabangana, 2019).

Comme conséquence de la dégradation biophysique, la ceinture périurbaine de Brazzaville, projette une matrice paysagère fortement anthropisée. Elle intègre la mosaïque forêt-savane du Congo-Ogooué, comme l'une des écorégions d'Afrique centrale forestière (White \& Vande weghe, 2008). Cependant, quelques rares îlots forestiers résiduels, se concentrent autour des grandes rivières, dont nombreuses sont des tributaires du fleuve Congo. Ces cordons ripicoles, bien occurrents en taxons relevant des Caesalpinioideae, sont interprétés comme des vestiges de la Dernière Glaciation Maximale du Pléistocène (Vincens et al., 2000 ; Ngomanda et al., 2009). Outre les forêts insulaires du fleuve Congo, conservées jusqu'à nos jours, en raison de leur incessibilité, entretenue par le régime hydrologique ; celles de la portion continentale, le sont grâce à une protection institutionnelle. Parmi ces derniers, on décèle la forêt refuge périurbaine de la Djoumouna, sous cotutelle de 1'Association des Scouts et Guides Catholiques du Congo (ASGC) et du Centre piscicole domanial. Ce site reflète une vitrine de la phytodiversité de la ceinture périurbaine de Brazzaville. Ce trait justifiait l'inscription cet habitat forestier parmi les vingt-trois sites critiques du pays par le Ministère des Eaux et Forêts le 25 juin 1974. Cette dernière, motivée suite à la sixième résolution prise au III ${ }^{\text {ème }}$ Congrès International du Fond Mondial pour la Nature (WWF), lors de sa réunion à Bonn le 5 octobre 1973.

En dehors de la forte représentativité de sa florule au sein de l'Herbier National (IEC), quelques travaux y sont répertoriés, en ethnobotanique (Miabangana \& Honjuila Miokono, 2015; 2016), en phytogéographie (Miabangana et al., 2016) et en phytosociologie (Miabangana, Op.cit).

Cependant ces données sus reprises, demeurent insuffisantes du fait qu'elles ne projettent pas toute la diversité floristique ; paramètre indispensable dans la détermination des indicateurs de la dynamique des phytocénoses et base incontournable dans l'aménagement forestier. De même de nombreux auteurs, tels Pitman et al. (2001) soulignent que la structure spatiale des espèces est l'un des paramètres clés en écologie pour la compréhension des processus écologiques et $\mathrm{du}$ fonctionnement des écosystèmes forestiers. Cristea (1997) abonde dans le même sens et souligne 
qu'on ne peut pas faire l'écophylaxie forestière, de l'évaluation de la qualité de l'environnement, de la conservation des zoocénoses, de la géomorphologie, de la gestion des réserves naturelles, etc. sans connaître la structure, la dynamique et le fonctionnement des phytocénoses.

La présente note se propose de caractériser la structure du peuplement forestier ; par l'approche du relevé polyvalent. Cette approche complémentaire de caractérisation écologique vient renforcer la connaissance de cette phytodiversité, en vue d'une gestion durable du site.

\section{Matériel et méthodes}

\section{Site d'étude}

La forêt de la Djoumouna est comprise entre les longitudes

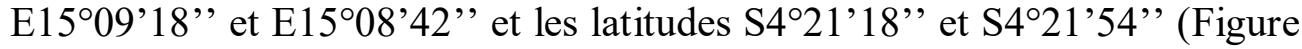
1). Elle est située dans le Département administratif du Pool, dans la souspréfecture de Goma tsé-tsé, et dans un vaste domaine de Yaka-Yaka. Ce domaine est situé à vingt-quatre kilomètres au sud-est de Brazzaville. Il est arrosé par un cours d'eau permanente, petit affluent du fleuve Congo, la Djoumouna qui prend sa source à Koubola, à une quinzaine de kilomètres en amont. Il est limité à l'est, par la rivière Djoumouna et l'un de ses affluents, la Maloto ; au sud, par la ferme piscicole de Djoumouna, la Ngarage et la Ngabankala dont les sources sont voisines ; à l'ouest par d'autres affluents de la Djoumouna et la route Nganga Lingolo-Linzolo.

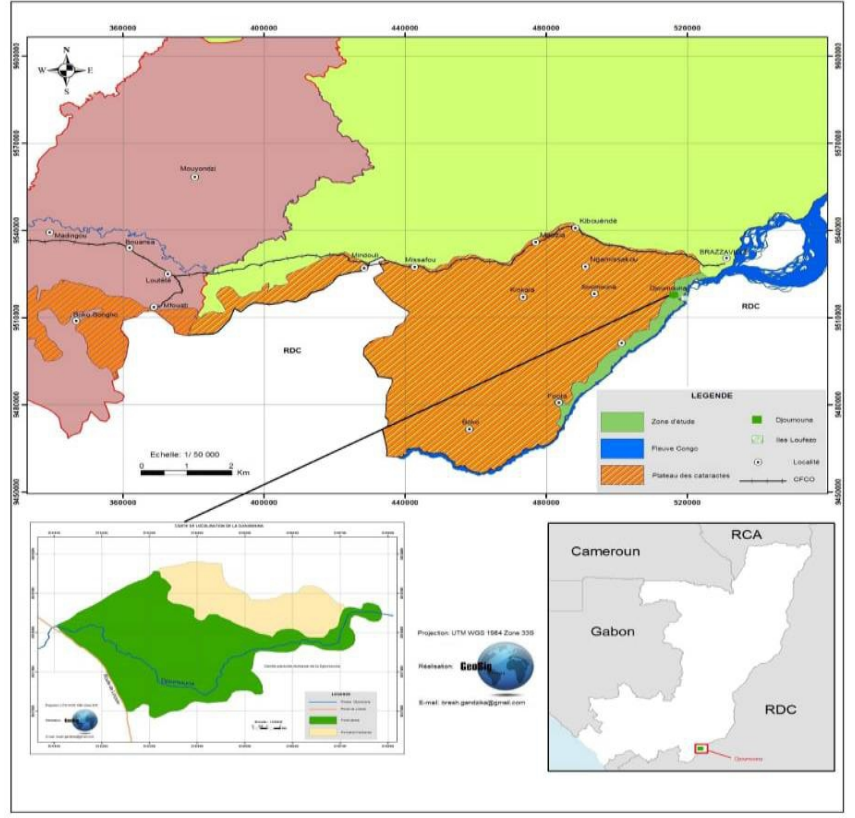

Figure 1. Localisation de la zone d'étude 
Géomorphologiquement le site de la Djoumouna est une pente qui fait suite au plateau de Yaka Yaka qui abritait l'ancienne chefferie traditionnelle. Ce site surplombe la rivière éponyme qui le parcourt sur près d'un kilomètre. La forêt occupe les deux flancs d'une vallée encaissée taillée par la rivière. Les rebords de la forêt présentent des pentes raides de près $30^{\circ}$ au niveau des cascades qui présentent par endroits de déclivités de 10 mètres le long de la rivière où affleure une dalle gréseuse. D'une longueur maximale de 620 mètres et d'une largeur correspondante de 270 mètres, la forêt couvre une superficie de 10,2 hectares.

Le climat est bas-congolais de type soudano-guinéen (Aubréville, 1949 ; Samba-Kimbata, 1978) qui, selon la classification de Köppen (1936) est de type AW4, c'est-à-dire caractérisé par une saison pluvieuse longue, entrecoupée par une saison sèche de trois à quatre mois, de juin à septembre.

Le climat est tropical humide, de type bas-congolais (Samba-Kimbata, 1978 ; Atlas 2001) qui, selon la classification de Köppen (1936) est de type $\mathrm{AW}_{4}$, c'est-à-dire caractérisé par une saison pluvieuse longue, entrecoupée par une saison sèche de trois à quatre mois, de juin à septembre.

Le substratum géologique est représenté essentiellement par des formations sédimentaires du Précambrien supérieur, de nature schistogréseuse où elles forment la série de l'Inkisi, et composé des arkoses et des grès feldspathiques, alors que les sols appartiennent à la classe des sols ferralitiques fortement désaturés remaniés jaunes (Denis, 1974).

$\mathrm{Au}$ niveau de la chorologie locale, le site est situé dans un écotone phytogéographique entre les sous-centres Congolais et Bas-Guinéen (Miabangana et al., Op cit), dans le Secteur de transition Bas-guinéozambézien et du District floristique du Plateau des Cataractes (Kimpouni et al., 1992).

\section{Méthodes de collecte et de traitement des données}

A partir d'un plan d'échantillonnage géoréférencé du site, de 10 hectares de superficie forestière, 25 relevés de végétation ont été exécutés, de février à août 2016. Ces relevés ont couvert $16 \%$ de la superficie forestière. Le tiers de la superficie du site, du côté de la rive droite de la rivière éponyme, est dominé par Bambusa vulgaris Schrad. ex J.C.Wendl., planté pour stabiliser le talus pour l'aménagement de la station aquacole domaniale, lors de la construction de la bretelle routière Nganga Lingolo-Linzolo, courant les années 1960.

Les mesures dendrométriques des espèces végétales, ont concerné les arbres et arbustes requérant une circonférence à hauteur de poitrine, CHP $\geq$ $31,4 \mathrm{~cm}$ (équivalent du diamètre à hauteur de poitrine, $\mathrm{dhp} \geq 10 \mathrm{~cm} \mathrm{ou} \mathrm{D}_{1,3} \geq$ $10 \mathrm{~cm}$ ). Ces dernières, ont tenu compte des ajustements avec les individus présentant des racines échasses, des contreforts et des cannelures 
(Pierlot,1966). Tous les autres ligneux, situés hors du relevé ont été également soumis à des mensurations et rattachés au relevé adjacent pour intégrer leur poids structural.

L'identification des espèces, amorcée in situ, a été complétée et confirmée à l'Herbier National du Congo (IEC), ceci par la comparaison entre les herbiers de référence et ceux conservés au sein de l'institution. La consultation des différentes Flores (Flore d'Afrique centrale, Flore du Gabon, Flore du Cameroun) et d'autres ouvrages, parfois fort bien illustrés (Tailfer, 1990 ; Hawthorne \& Jongkind, 2006 ; Hawthorne \& Gyakary, 2006 ; Harris \& Wortley, 2008 ; Meunier et al., 2015 ; Vande weghe et al., 2016) ; a appuyé permis de conforter les déterminations botaniques.

La consultation de cette documentation scientifique, nous a encore permis de disposer de l'identification des caractéristiques écologiques des espèces ainsi que leurs distributions phytogéographiques.

La nomenclature adoptée suit l'APG IV (2016), renforcée par le référentiel taxonomique de Lebrun et Stork (1991-2015) et accessible sur le site régulièrement mis à jour.

\section{Analyse des données}

\section{Paramètres de caractérisation botanique}

Ces derniers concernent la diversité des familles (DiF), la surface terrière relative $\left(\mathrm{St}_{\mathrm{r}}\right)$, la densité relative $\left(\mathrm{D}_{\text {rel }}\right)$, la dominance relative $\left(\mathrm{Do}_{\text {rel }}\right)$, la fréquence relative $\left(\mathrm{F}_{\text {rel }}\right)$, l'indice de Valeur d'Importance des familles (FIV) et l'indice de valeur d'importance relative des espèces ( $\left(\mathrm{VI}_{\mathrm{rel}}\right)$.

La diversité des familles

La surface terrière relative

$$
\mathrm{DiF}=\frac{\text { Nombre } \mathrm{d}^{\prime} \text { espèces de la famille } \mathrm{F} \times 100}{\text { Nombre total } \mathrm{d}^{\prime} \text { espèces }}
$$

$$
\mathrm{St}_{\text {rel }}=\sum_{\mathrm{i}=1}^{\mathrm{n}} \frac{\pi \mathrm{Di}^{2}}{4}
$$

$\mathbf{S t}_{\mathrm{r}}$ : surface terrière relative, exprimée en $\mathrm{m}^{2} / \mathrm{ha} ; \mathrm{Di}$ : diamètre à $1,3 \mathrm{~m}$ du sol de l'arbre $\mathrm{i} ; \mathrm{n}$ : nombre total d'individus de l'espèce. La surface terrière totale $\left(\mathrm{St}_{\mathrm{t}}\right)$ correspond à la somme des surfaces terrières de tous les individus de la composition floristique, présents sur la surface inventoriée.

La densité relative $\left(\mathrm{D}_{\text {rel }}\right)$

$$
\mathrm{D}_{\text {rel }}=\frac{\text { Nombre } \mathrm{d}^{\prime} \text { individusde } \mathrm{l}^{\prime} \text { espèce } \times 100}{\text { Nombre total } \mathrm{d}^{\prime} \text { individus de toutes les espèces recensées }}
$$

La dominance relative $\left(\mathrm{Do}_{\text {rel }}\right)$

$$
\text { Do }_{r e l}=\frac{\text { Surface terrière de l' espèce } \times 100}{\text { Somme des surfaces terrières de toutes les espèces }}
$$

La fréquence relative $\left(\mathrm{F}_{\text {rel }}\right)$ 


$$
\mathrm{F}_{\text {rel }}=\frac{\text { Fréquence } \mathrm{d}^{\prime} \text { une espèce } \mathrm{x} 100}{\text { Somme des fréquences de toutes les espèces }}
$$

La fréquence d'une espèce correspond au nombre d'occurrences contenant cette espèce dans une unité d'échantillonnage.

L'indice de Valeur d'Importance des Familles (Family Importance Value : FIV)

$$
\mathrm{FIV}=\mathrm{D}_{r e l}+\mathrm{Do}_{r e l}+\mathrm{DiF}
$$

Utilisé par Nusbaumer et al. (2005), cet indice fournit des informations sur l'importance floristique de chaque famille ainsi que son éventuel potentiel structurant au sein du peuplement.

L'indice de valeur d'importance relative $\left(\mathrm{IVI}_{\mathrm{r}}\right)$

$$
\mathrm{IVIr}=\mathrm{D}_{\text {rel }}+\mathrm{Do}_{\text {rel }}+\mathrm{F}_{\text {rel }}
$$

Ce dernier indice permet de déterminer les espèces les plus dominantes dans l'aire d'échantillonnage. Il est dérivé de l'Importance Value Index de Curtis et Mc Intosh (1951) et repris par quelques auteurs en Afrique Équatoriale Atlantique (Reitsma, 1988 ; Lejoly, 1993 ; Doucet, 2003). Ainsi les espèces les plus dominants (Leading dominant) sont celles qui réalisent les plus grandes valeurs d'IVIr.

Enfin, afin d'évaluer le degré de maturité et de stabilité de la florule forestière, nous avons calculé le quotient spécifique de Szymkiewicz (Evrard, 1968), noté $\mathrm{I}$ et formulé par: $\mathrm{I}=\mathrm{Ge} / \mathrm{S}$; où $\mathrm{S}$ est le nombre d'espèces identifiées dans chaque forêt et Ge le nombre de genres.

\section{Les indices de diversité}

Quelques indices de diversité ont été calculés : la richesse et la diversité floristiques, les indices de diversité de Shannon et Weaver $\left(\mathrm{H}^{\prime}\right)$, de Simpson (D) et de l'indice de richesse de Margalef $\left(\mathrm{D}_{\mathrm{Mg}}\right)$ et enfin l'indice d'équitabilité de Piélou (E).

La richesse et la diversité spécifiques

La richesse spécifique est le nombre total d'espèces $(\mathrm{S})$ rencontrées dans un peuplement. La diversité spécifique quant à elle renvoie à la répartition de l'effectif total $(\mathrm{N})$ entre les différentes espèces.

L'indice de Shannon et Weaver (H')

$$
\mathrm{H}^{\prime}=-\sum \mathrm{Pi} \ln \mathrm{Pi}
$$

où $\mathrm{P}_{\mathrm{i}}$ est l'abondance relative de l'espèce $\mathrm{i}(\mathrm{Pi}=\mathrm{ni} / \mathrm{N}), \mathrm{ni}=$ nombre d'individus par espèce; $\mathrm{N}=$ nombre d'individus total par unité d'échantillonnage. $\mathrm{H}$ varie de 0 à 5 bits, voire un peu plus de 5 . Une valeur élevée de cet indice traduit des conditions stationnelles favorables à l'installation de nombreuses espèces ; c'est l'expression d'une grande stabilité du milieu (Dajoz, 1996).

L'indice de Simpson (D)

$$
\mathbf{D}=\sum(\mathrm{Pi})^{2}
$$


L'indice de Simpson représente la probabilité pour que deux individus pris au hasard dans le peuplement étudié appartiennent à la même espèce. Il mesure la manière avec laquelle les individus se répartissent entre les espèces d'une communauté. Sa valeur tend vers 0 pour indiquer le maximum de diversité et vers 1 pour indiquer le minimum de diversité.

L'indice de Margalef $\left(\mathrm{R}_{\mathrm{Mg}}\right)$

$$
\mathbf{R}_{\mathbf{M g}}=\frac{\mathbf{S}-1}{\operatorname{Ln}(\mathbf{N})}
$$

La valeur de l'indice de Margalef renseigne si la richesse spécifique d'une communauté végétale est élevée ou non. Indice à comparer avec des données ultérieures provenant d'autres portions d'écosystèmes.

L'équitabilité de Piélou (1966)

$$
\mathbf{E}=\frac{\mathrm{H}^{\prime}}{\operatorname{Ln} \mathrm{S}}=\frac{\mathrm{H}^{\prime}}{\mathrm{Hmax}}
$$

En ce qui concerne l'équitabilité de Piélou, sa valeur varie de 0 à 1 . La valeur élevée est un indicateur d'un peuplement équilibré (Dajoz, Op cit). Les intervalles des valeurs mentionnées par Inoussa et al. (2013) permettent d'apprécier le degré d'équitabilité d'une phytocénose.

\section{Résultats et discussion}

\section{Caractéristiques floristiques}

Nos investigations de terrain, provenant de 1039 individus requérant un $\mathrm{dbh} \geq 10 \mathrm{~cm}$, livrent une richesse floristique de 123 espèces et taxons infra spécifiques ; les noms d'auteurs étant repris en Annexe 1. Ces dernières s'organisent en 104 genres et 41 familles. La valeur du quotient spécifique de la florule inventoriée est de 0,84 . Les familles les plus diversifiées (totalisant au moins 8 espèces), se recrutent parmi les Leguminosae (Fabaceae), les Euphorbiaceae et les Rubiaceae. La Figure 2 présente la diversité de 14 principales familles de la matrice florale. Les genres Dialium, Millettia et Oncoba sont les plus diversifiés, renfermant tous trois, trois espèces.

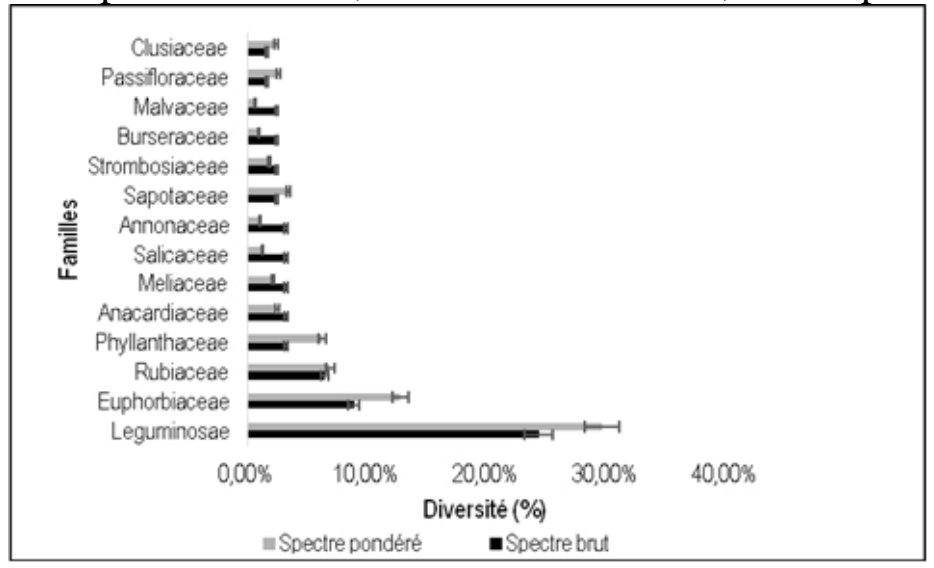

Figure 2. Diversité des familles les plus représentées 
Le fond floristique des individus requérant un $D_{1,3} \geq 10 \mathrm{~cm}$ est relativement riche et diversifié dans le peuplement forestier. Elle représente $27 \%$ de la florule totale, ceci en comparaison avec les données d'inventaire du site (Miabangana et al., 2016). La tendance floristique, concernant la diversité des familles, demeure la même. Ce fond reste dominé par les Caesalpinioideae, sous famille très ancienne. Cette tendance est aussi relevée par White (1986) pour les forêts denses d'Afrique. Observations relayées dans les investigations menées dans les sous centres Bas-guinéen (Sonké, 1998 ; Doucet, 2003 ; Campbell et al., 2006) et congolais (Anani et al., 2013 ; Lisingo et al., 2015). Ce trait confirme de facto la position de refuge de ce cordon ripicole (Miabangana, 2019). La valeur quotient spécifique $(0,81 \leq 1)$ relativement basse et inférieure à celle de la florule totale (Miabangana et al., op cit). Cette dernière étant de 1,5 ; traduit la maturité et la stabilité de la florule idoine. Cette valeur reflète les mêmes tendances dans les travaux de nombreux auteurs, tels Lebrun (1960), Evrard (1968) et Sonké (Op. cit). Les familles des Euphorbiaceae et des Rubiaceae qui se relaient aux Leguminosae sont également signalées par les auteurs sus repris, ainsi que dans des investigations menées dans le sous-centre de la Haute Guinée (Guillaumet, 1967 ; N'da et al., 2008 ; Nusbaumer et al., Op. cit).

\section{Spectres biologiques}

Seul le type phanérophytique est retenu dans cet inventaire. La catégorie mésophanérophytique ( $\mathrm{MsPh})$, se recrutant parmi les individus de la canopée, est prépondérante (Figure 3).

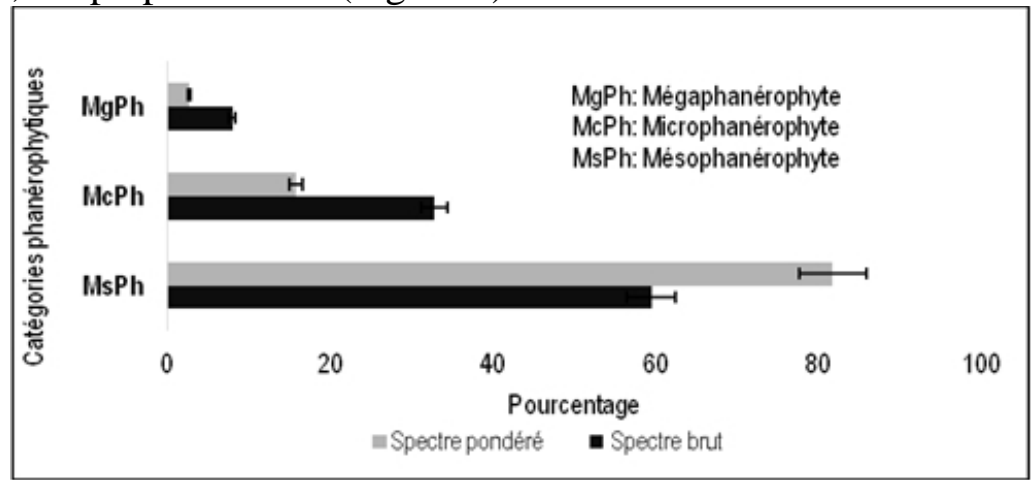

Figure 3. Spectres des catégories phanérophytiques de la forule

Dans ces catégories phanérophytiques, les mégaphanérophytes se recrutent parmi les arbres émergents tels, Anopyxis klaineana, Ceiba pentandra., Daniellia klainei, Gilbertiodendron dewevrei, Lannea welwitschii, Pseudospondias microcarpa, Psydrax arnoldiana et Uapaca guineensis. En dehors des trois premières espèces, les autres sont également distribuées dans le type mésophanérophytes structurant la canopée. Quant aux 
microphanérophytes, tels Alchornea floribunda, Carapa procera, Chaetocarpus africanus, Cola heterophylla, Oddoniodendron romeroi et Oxyanthus speciosus, ils structurent la synusie arbustive.

\section{Spectres phytogéographiques}

En se référant à la classification de White (1986), la matrice florale reste largement dominée par les espèces guinéo-congolaises, telles que reprises à la Figure 4.

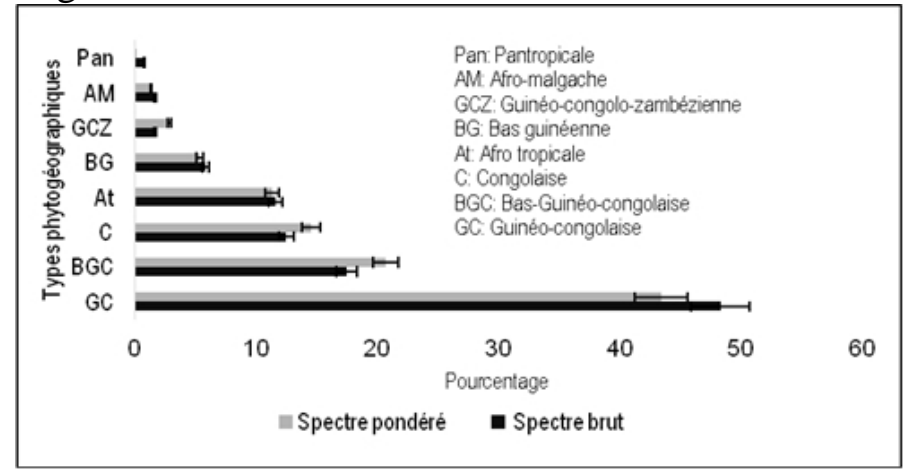

Figure 4. Spectres des types phytogéographiques de la florule

Ces espèces pluri sous centres (GC), associées aux bas guinéocongolaises et aux congolaises confortent le caractère endémique (EE) de la souche florale (Figure 5).

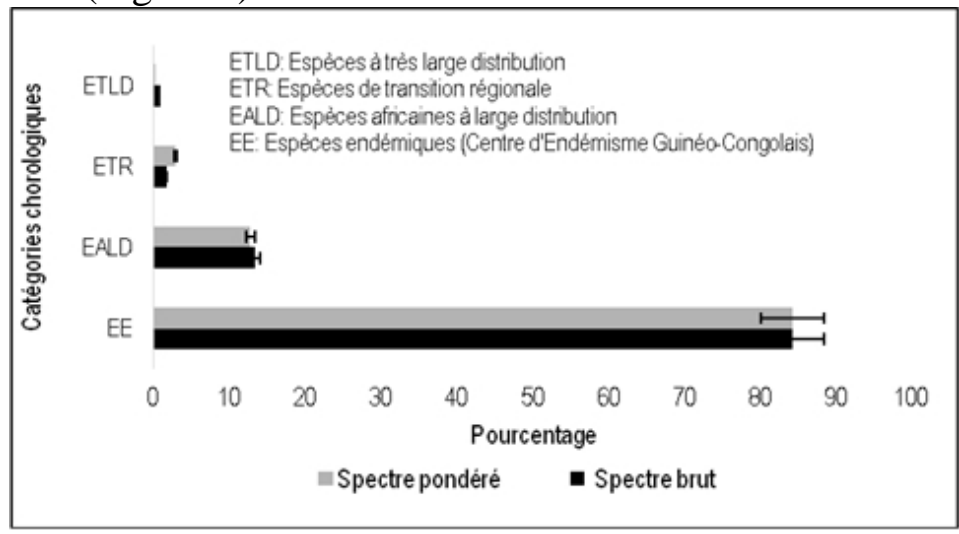

Figure 5. Catégories chorologiques de la florule

Les spectres phytogéographiques confirment l'originalité de cette florule, en dépassant légèrement la prévision de White (1986). En effet, ce dernier considère que la flore guinéo-congolaise est remarquablement pure, avec plus de $80 \%$ d'endémiques et seulement environ $10 \%$ d'espèces de liaison. Les proportions de $1,6 \%$ et de $2,9 \%$ des spectres brut et pondéré que réalisent les espèces de transition régionale dans notre matrice florale, confirme la faible altération de ce site. 


\section{Caractéristiques structurales}

\section{Densité, structure diamétrique et surface terrière}

Sur une superficie de $15.900 \mathrm{~m}^{2}$ (1,59 ha), émanant des 25 relevés de végétation, 1039 individus ont été recensés (soit 653 individus/ha). Ces individus; se recrutant largement parmi les arbres; se répartissent en six classes de diamètre. La courbe à l'allure exponentielle descendante, montre que le nombre d'individus décroît significativement avec l'augmentation du diamètre des arbres. La Figure 6 reprend la structure diamétrique du peuplement.

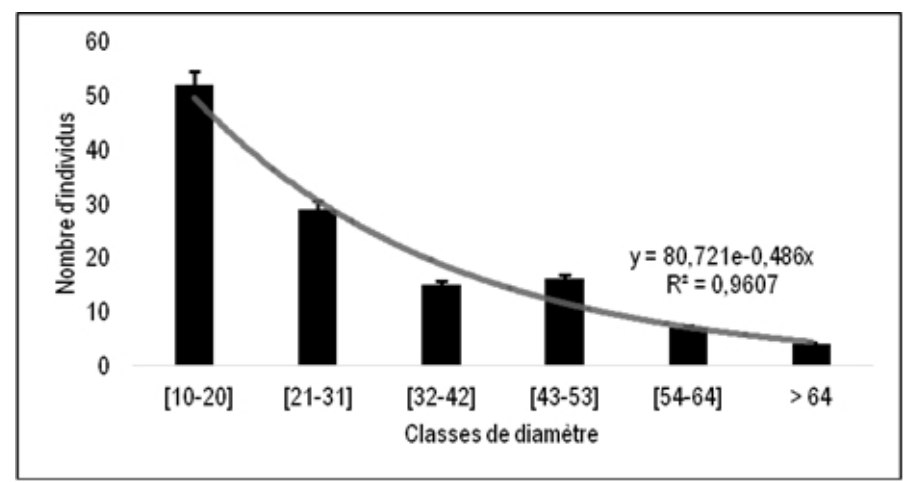

Figure 6. Structure diamétrique du peuplement forestier

Quant à la surface terrière, tout le peuplement forestier réalise une valeur basale de $28 \mathrm{~m}^{2} /$ ha, dont $18 \mathrm{~m}^{2} /$ ha, soit $66 \%$ reviennent à la seule famille des Leguminosae.

L'allure exponentielle descendante de notre distribution diamétrique est similaire aux observations de nombreux auteurs, tels, Pascal, Op cit ; Dupuy, 1998 ; Anani et al., 2013 ;_Omatoko, 2015 ; Kambale et al., 2016 et Tsoumou et al., 2016. Ces faits confirment que notre peuplement forestier affiche un potentiel de régénération, en dynamique progressive (sensu Hallé et al., 1978). Ce qui ouvre une orientation sur la capacité de séquestration de carbone de ce site périurbain.

Prises isolément, certaines espèces, malgré des occurrences d'abondance faibles au sein de ce peuplement, affichent des dbh élevés. Le Tableau 1 reprend les arbres qui présentent des fortes valeurs de dbh.

Tableau 1. Espèces aux individus de $D_{1,3} \geq 60 \mathrm{~cm}$

\begin{tabular}{|c|c|c|c|c|c|c|}
\hline \multirow[t]{2}{*}{ Espèces végétales } & \multicolumn{6}{|c|}{ Valeurs de Dbh $(\mathrm{cm})$} \\
\hline & 120 & 95 & 80 & 70 & 65 & 60 \\
\hline $\begin{array}{l}\text { Crudia laurentii * } \\
\text { Irvingia grandifolia } *\end{array}$ & $\begin{array}{l}X \\
X\end{array}$ & & & & & \\
\hline Quassia silvestris & & $\bar{X}$ & & & & \\
\hline $\begin{array}{l}\text { Chrysophyllum subnudum } \\
\text { Cynometra sessiliflora } \\
\text { Gilbertiodendron dewevrei }\end{array}$ & & & $\begin{array}{l}X \\
X \\
X\end{array}$ & & & \\
\hline
\end{tabular}




\begin{tabular}{|c|c|c|c|c|}
\hline $\begin{array}{l}\text { Hymenostegia neoaubrevillei } \\
\text { Paramacrolobium coeruleum } \\
\text { Pentaclethra eetveldeana } \\
\text { Pentaclethra macrophylla }\end{array}$ & $\begin{array}{l}X \\
X \\
X \\
X\end{array}$ & & & \\
\hline $\begin{array}{c}\text { Celtis tessmannii } \\
\text { Dacryodes pubescens } \\
\text { Millettia laurentii } \\
\end{array}$ & & $\begin{array}{l}X \\
X \\
X \\
\end{array}$ & & \\
\hline $\begin{array}{c}\text { Albizia ferruginea } \\
\text { Anopyxis klaineana* } \\
\text { Daniellia klainei * } \\
\text { Pseudospondias microcarpa } \\
\text { Uapaca guineensis }\end{array}$ & & & $\begin{array}{l}X \\
X \\
X \\
X \\
X\end{array}$ & \\
\hline $\begin{array}{c}\text { Aphanocalyx microphyllus subsp. Microphyllus } \\
\text { Dracaena arborea* } \\
\text { Manilkara aubrevillei } \\
\text { Musanga cecropioïdes } \\
\text { Petersianthus macrocarpus } \\
\text { Psydrax arnoldiana }\end{array}$ & & & & $\begin{array}{l}X \\
X \\
X \\
X \\
X \\
X\end{array}$ \\
\hline
\end{tabular}

* : Un seul individu de l'espèce présent dans le peuplement ; X : Présent.

A l'échelle spécifique, ce sont Pentaclethra eetveldeana et Pentaclethra macrophylla qui présentent les valeurs les plus élevées de surface terrière, en réalisant respectivement 11 et $05 \mathrm{~m}^{2} /$ ha dans ce peuplement. La Figure 7 illustre les 9 espèces aux valeurs de surfaces terrières les plus élevées.

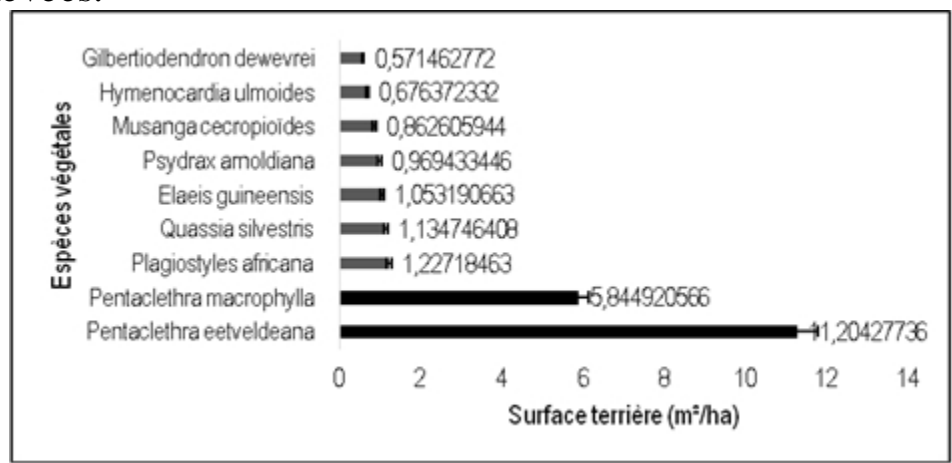

Figure 7. Espèces réalisant les valeurs les plus élevées en surface terrière

En ce qui concerne les aspects structuraux, nous comparons nos résultats sur la densité et la surface terrière moyenne, aux observations de Reitsma (1998) au Gabon ; de Nhsimba (2008), de Loris-Lukens (2009), de Menga (2012) et d'Omatoko (2015) respectivement sur l'île Mbiye à Kisangani, dans les forêts denses à Masako à Kisangani, au Mai Ndombé et dans la forêt de plaine d'Uma dans le district de la Tshopo (Tableau 2). Toutes les dernières localités sont situées en République Démocratique du Congo. 
Tableau 2. Comparaison de la densité et de la surface terrière de la Djoumouna avec Les données des forêts équatoriales

\begin{tabular}{|c|c|c|}
\hline Auteurs & Densité (Tiges/ha) & Surface terrière $\left(\mathrm{m}^{2} / \mathrm{ha}\right)$ \\
\hline Présente étude & 653 & 28 \\
Reitsma (1998) & 435 & 35,7 \\
Nshimba (2008) & 507 & 21,15 \\
Loris-Lukens (2009) & 469 & 30,73 \\
Menga (2012) & 364 & $/$ \\
\hline Omatoko (2015) Sol argileux (Pericopsis) & 467 & 29 \\
Omatoko (2015) Sol sableux (Julbernardia) & 344 & 24 \\
\hline
\end{tabular}

L'analyse de ce tableau montre que notre peuplement présente une densité très élevée, par rapport aux autres travaux similaires ; ceci malgré le climat tropical humide auquel est soumis notre site. Cette valeur intègre la prévision de Rollet (1983) et de Pascal (2003). Ces derniers mentionnent que pour un diamètre minimal de $10 \mathrm{~cm}$, la densité des tiges à l'hectare varie entre 450 et 750 individus, prouvant que l'îlot forestier ne présente pas de contraintes particulières.

La valeur de la surface terrière par hectare de notre groupement est inférieure à la moyenne de de Reitsma, de Loris-Lukens (Op-cit) et légèrement de d'Omatoko (Op-cit), sur substrat argileux. Le gradient climatique et les caractéristiques édaphiques locales sont autant de paramètres justificatifs. Tout de même, notre valeur intègre sans ambiguité la prévision de Pascal (Opcit). Ce dernier affiche une marge de 25 et $50 \mathrm{~m}^{2} / \mathrm{ha}$ pour les forêts denses tropicales humides. Cette valeur approche de même la prédiction de Doucet (2003) qui la situe dans la fourchette de 30 et $35 \mathrm{~m}^{2} /$ ha pour les forêts tropicales africaines.

\section{Structure verticale}

Six classes de hauteurs ont également été décelées dans ce peuplement forestier. Les individus dont la classe de hauteur varie entre 17 et 22 mètres sont les plus représentatifs, à hauteur de 37,4\%. La Figure 8 mentionne la structuration verticale du peuplement.

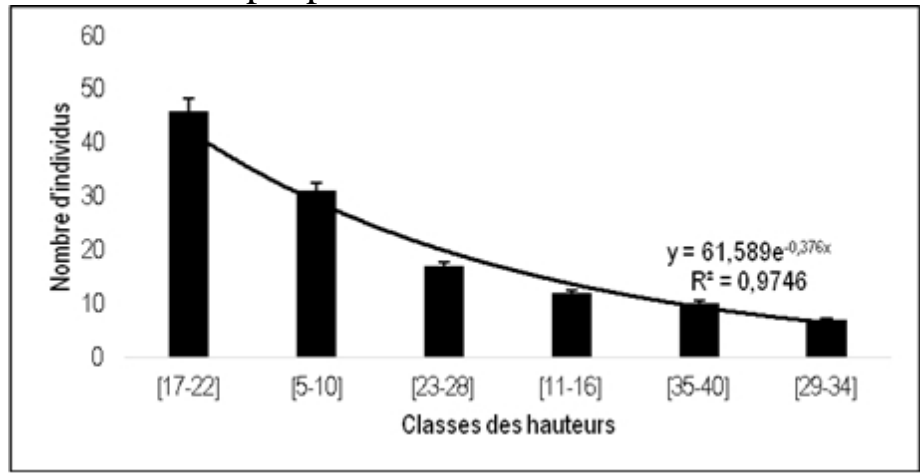

Figure 8. Structure verticale du peuplement 
La Figure 9 présente la distribution spatiale de tous les arbres géoréférencés de $D_{1,3} \geq 50 \mathrm{~cm}$ du peuplement forestier.

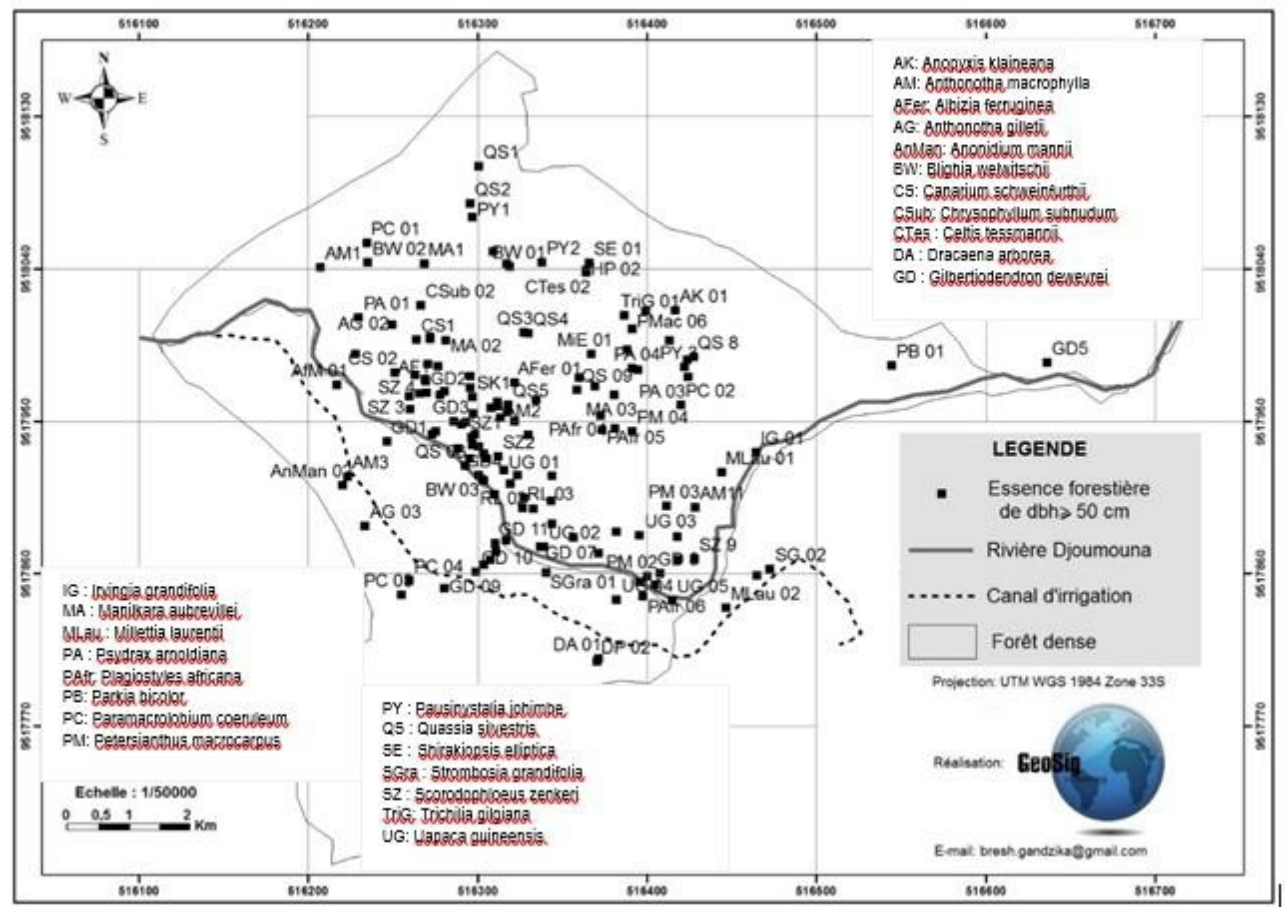

Figure 9. Distribution des arbres de $D b h \geq 50 \mathrm{~cm}$

\section{Valeur d'importance des taxons}

En ce qui concerne les familles, ce sont les Leguminosae qui réalisent la valeur la plus élevée de l'indice d'importance des familles (FIV, Family Iimportance Value). Ces dernières sont relayées respectivement par les Euphorbiaceae, les Rubiaceae et les Phyllanthaceae. La Figure 10 présentent les 11 familles les plus représentatives du peuplement forestier. 


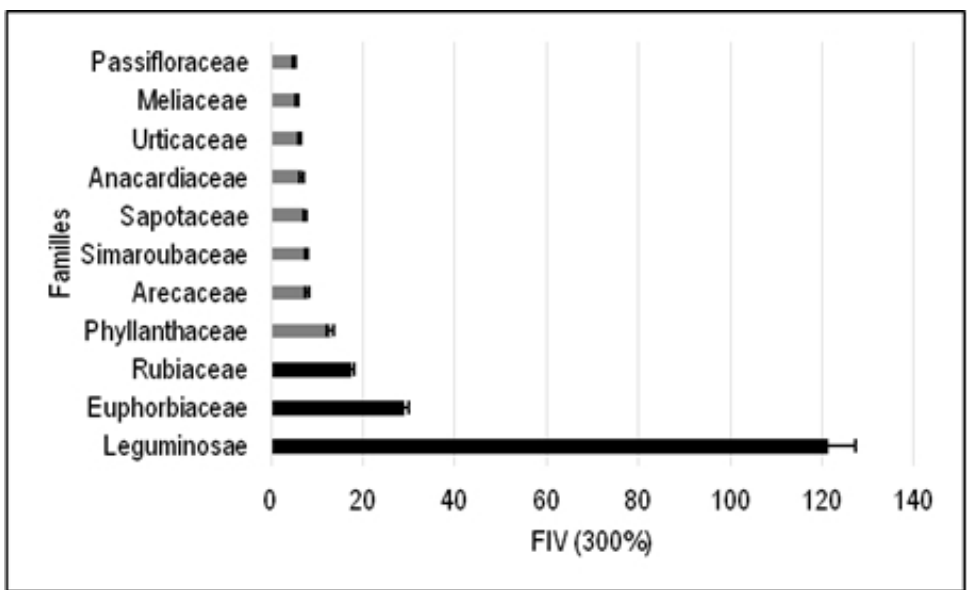

Figure 10. FVI des familles prépondérantes du peuplement forestier

Quant au niveau spécifique, ce sont Pentaclethra eetveldeana et Pentaclethra macrophylla qui confirment les valeurs les plus élevées, en Indice de Valeur d'Importance (Importance Value Index, IVI). La Figure 11 illustre les 13 taxons les plus représentatifs de ce paramètre.

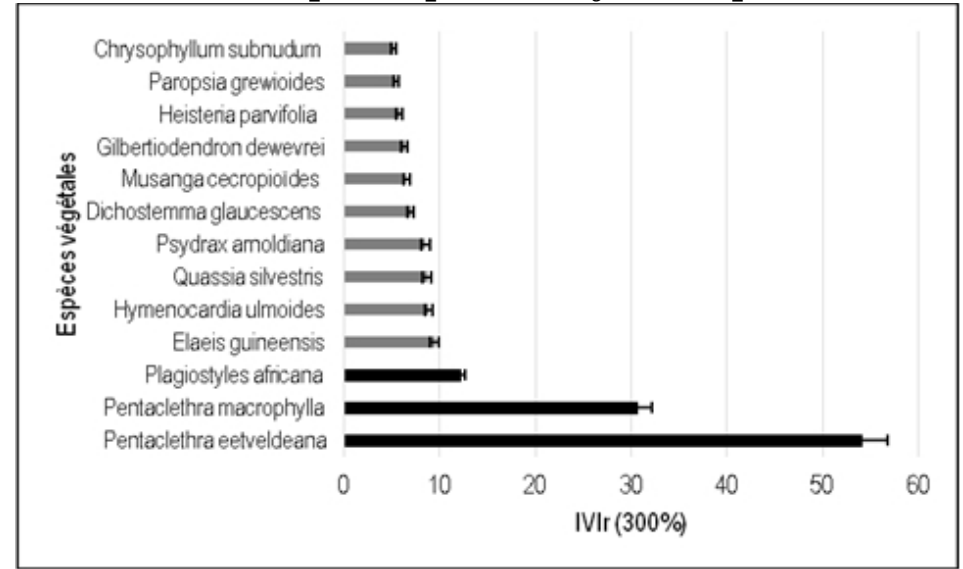

Figure 11. Espèces aux valeurs d'IVI les plus élevées

En ce qui concerne l'indice d'importance des taxons, la prépondérance écologique des Leguminosae, des Euphorbiaceae et des Rubiaceae. Nusbaumer et al. (Op. cit), mentionnent les mêmes observations dans la forêt classée du Scio, en Côte d'Ivoire. La dominance des Leguminosae dans notre florule n'est guère surprenante, en comparant nos investigations à celles rapportées quelques auteurs (Nusbaumer et al., Op-cit. ; Campbell et al., 2006 ; Omatoko et al., 2015 ; Kambale et al., 2016). L'importance écologique des Leguminosae est confortée par la sous famille des Caesalpinioideae en spectre brut et celle des Mimisoideae, en spectre pondéré, plus précisément avec le genre Pentaclethra Benth. A l'échelle spécifique, ce sont respectivement Pentaclethra eetveldeana, Pentaclethra macrophylla et Plagiostyles africana 
qui projettent l'armature structurale dans le peuplement ligneux, d'après la prévision de Reitsma (Op-cit) qui fixe le seuil à la valeur d'IVIr $\geq 10$. Le genre Pentaclethra ne renferme que ces deux idiotaxons, sus repris, dans la flore du Congo (Sita \& Moutsamboté, 1988). Ce sont des espèces longévives de forêts secondaires, de diaspores de type ballochore et de dissémination autochore, recélant un grand potentiel séminal advectif. Ce trait justifie sa dénomination structurale de forêt à Pentaclethra Benth. (Leading dominant).

Les indices de diversité du peuplement ligneux sont repris dans le Tableau 3.

Tableau 3. Indices de diversité du peuplement forestier

\begin{tabular}{|c|c|}
\hline Shannon et Weaver (H') & 6,94601 \\
\hline Simpson $(\mathrm{D})$ & 0,00096 \\
\hline 1-D & 0,99904 \\
\hline Equitabilité de Piélou (E) & 1,4 \\
\hline Margalef $\left(\mathrm{R}_{\mathrm{Mg}}\right)$ & 17,56 \\
\hline
\end{tabular}

Ces indices projettent une bonne diversité du peuplement forestier et une équitabilité élevée. Quant à l'indice de Margalef, il permettra par la suite de comparer la richesse floristique du site avec d'autres portions d'écosystèmes forestiers dans le Plateau des Cataractes.

\section{Comment comprendre une bonne structuration du peuplement forestier de la Djoumouna, comparable à celle des forêts équatoriales, dans une écorégion de mosaïques forêts-savanes ?}

Trois arguments d'importance inégale méritent d'être envisagés : paléoclimatique, écologique et anthropique. Des travaux paléoclimatiques mentionnent que l'écorégion de la mosaïque forêt-savane du Congo-Ogooué (White \& Vande weghe, Op-cit), était occupée par des forêts denses jusqu'avant la Grande Glaciation Maximale du Pléistocène (Vincens et al., 2000 ; Leal, 2004 ; Ngomanda et al., 2009). La matrice paysagère actuelle est interprétée comme un héritage de la phase post péjoration climatique. Certains îlots forestiers, en position de refuge, auraient résisté aux températures extrêmes en raison de la proximité d'un cours d'eau. Le réseau hydrographique, de par l'humidité édaphique, agissait comme un 'système tampon'" pour le maintien de ces îlots dont le fond floristique est dominé par les Caesalpinioideae. Le deuxième argument se focalise sur la "'compensation écologique'. En effet les conditions édaphiques sus évoquées, et physiographiques favorables ont permis la recolonisation forestière à l'Holocène, avec une occurrence d'espèces de forêts ombrophiles équatoriales, dans les zones où règnent les forêts semi-caducifoliées (Lebrun \& Gilbert, 1954). Enfin, à défaut d'un classement, le cordon ripicole de la Djoumouna, est depuis plus d'une soixante d'année, sous cotutelle de l'Église Catholique et des services des Eaux et Forêts. Ces deux institutions veillent à 
la conservation de cette portion d'écosystème qui l'épargne d'une grande empreinte humaine.

\section{Conclusion}

Cette étude, qui s'est focalisée sur la connaissance de la végétation forestière de la Djoumouna. L'approche méthodologique, étant celle du relevé polyvalent qui s'est relayée à la caractérisation phytosociologique.1039 individus ligneux de $D_{1,3} \geq 10 \mathrm{~cm}$, ont été soumis à des mensurations dendrométriques. 25 relevés de végétation, totalisant 1,59 ha de superficie, ont été exécutés. Ce peuplement ligneux, bien diversifié et intégré à la souche florale guinéo-congolaise ; livre une richesse floristique de 123 espèces. Ces dernières se répartissent en en 104 genres et 41 familles. Le fond floristique étant essentiellement dominé par les Leguminosae. Deux espèces imposent leur poids structural au peuplement forestier: Pentaclethra eetveldeana et Pentaclethra macrophylla.

Trois déterminants ont favorisé la bonne structuration de site forestier : paléoclimatique, écologique et une faible empreinte humaine. Toute fois la capacité de séquestration de carbone de ce peuplement ligneux, mérite d'être abordée, aux fins d'affiner la base scientifique d'un aménagement à titre conservatoire de ce miroir de la biodiversité périurbaine.

\section{References:}

1. Amani, A.C., Milenge, K.H., Lisingo, J., \& Nshimba, H. (2013). Analyse floristique et impact du déterminisme édaphique sur l'organisation de la végétation dans les forêts de l'ile Kongolo (R. D. Congo). Geo-Eco-Trop., 37, 2 : 255-272.

2. APG IV (2016). An updated of the Angiosperm Phylogeny Group classifications for ordersand families of flowering plants: APG IV (PDF). Botanical Journal of the Linnean Society181(1): 1-20. doi :10.1111/boj.12385.ATLAS (2001). Congo. Les Atlas d'Afrique, éditions Jeune Afrique, Paris : 76 p

3. Aubréville, A. (1949). Ancienneté de la destruction de la couverture florestière primitive de l'Afrique tropicale (Conf. Afric. Sols 1948, Goma). Bull. Agric. Congo-Belge, vol. XL, $\mathrm{n}^{\circ} 2$ :

4. 1347-1352.

5. Campbell, P., Rivera, P., Thomas, D., Bourobou-Bourobou, H., Nzabi, Th., Alonso, A., \& Dallmeier, F. (2006). Structure, composition et diversité floristiques d'une forêt équatoriale du Gabon. Bulletin of the Biological Society of Washington, No.12 : 29-52.

6. Curtis, J.T. \& McIntosh, R.P. (1951). An upland forest continuum in the prairie-forest border region of Wisconsin. Ecology 32 : 476-496.

7. Dajoz, R. (1996). Précis d'écologie. DUNOD. 551p. 
8. Denis, B. (1974). Carte pédologique Brazzaville-Kinkala. Notice explicative $n^{\circ 52}$, République Populaire du Congo à 1.200.000. Paris, ORSTOM.

9. Doucet, J.L. (2003). L'alliance délicate de la gestion forestière et de la biodiversité dans les forêts du Centre du Gabon. Thèse de doctorat, Faculté Universitaire des Sciences Agronomiques de Gembloux, 323 p.

10. Dupuy, B. (1998). Bases pour une sylviculture en forêt dense humide africaine. Série RORAFI 1998, document 4 CIRAD-Forêt, Montpellier, $328 \mathrm{p}$.

11. Evrard, C. (1968). Recherches écologiques sur le peuplement forestier des sols hydromorphes de la Cuvette Centrale Congolaise. INEAC, série scientifique $\mathrm{n}^{\circ} 110$.

12. Guillaumet, J.L. (1967). Recherches sur la végétation du Bas-Cavally (Côte d'Ivoire). Office de la Recherche Scientifique et Technique d'Outre-Mer, ORSTOM Paris, 247p.

13. Hallé, F., Oldeman, A. A., \& Tomlinson, P. B. (1978). Tropical trees and forests. An architectural analysis. Springer Verlag, Berlin, Heidelberg, New-York, $441 \mathrm{p}$.

14. Harris, D.J. \& Wortley, A.H. (2008). Les arbres de la Sangha. Manuel d'Identification illustré. Traduction française de Frachon, N. Royal Botanic Garden, Edinburg, United Kingdom : 300 p.

15. Hawthorne, S.W. \& Gyakary, N. (2006). Photoguide for the Forest Trees of Ghana. A treespotter's field guide for indentifying the largest trees. Oxford Forestery Institute, Dpt ofPlant Sciences, UK : 432 p.

16. Hawthorne, S.W. \& Jongkind, C. (2006). Woody plants of western African forest. A guide to the forest trees, shrubs and lianes from Senegal to Ghana. Kew, Roy. Bot. Gardens, Kew: 1023 p.

17. Inoussa, T.M., Imourou, I.T., Gbègbo, M.C., \& Sinsin, B. (2013). Structure et composition floristiques des forêts denses sèches de la région des Monts Kouffé au Bénin. Journal of Applied Biosciences 67 : 4787-4796.

18. Kambale, J.L.K., Shutsha, R. E., Katembo, E.W., Omatoko, J.M., Kirongozi, F.B., Basa, O.D., Bugentho, E.P., Yokana, E.I., Bukasa, K.K., Nshimba, H.S., \& Nyiwa Ngbolua, K. (2016). Etude floristique et structurale de deux groupements végétaux mixtes sur terre hydromorphe et ferme de la forêt de Kponyo (Province du Bas-Uélé, R.D. Congo). International Journal of Innovation and Scientific Research. Vol. 24 No. 2 Jun. pp. 300-308.

19. Kimpouni, V., Lejoly, J., \& Lisowski, S. (1992). Les Euriocaulaceae du Congo., Frang, Flor.Geobot. 37 (1) 127 - 145. 
20. Köppen, W. (1936). Das geographische system der klimate. In Köppen, W. et Geiger, R(eds.) Handbuch der klimatologie : 1-44. Berlin.

21. Leal, M.E. (2004). The African rain forest during the Last Glacial Maximum, an archipelago of forests in a sea of grass. Ph.D thesis Wageningen University, Wageningen: $110 \mathrm{p}$.

22. Lebrun, J. (1960). Sur une méthode de délimitation des horizons et étages de végétation de montagne Au Congo oriental. Bull. Jard. Bot. Etat, Bruxelles, 30 : 75-94.

23. Lebrun, J.P. \& Stork, A.L. (1991-2015). Enumération des plantes à fleurs d'Afrique tropicale. Conservatoire et Jardin botaniques de la Ville de Genève, 4 volumes.

24. http://www.villege.ch/musinfo/bd/cjb/africa/recherche.php?langue=fr

25. Lejoly, J. (1993). Méthodologie pour les inventaires forestiers (Partie flore et végétation). AGRECO-CTFT, Bruxelles, 53 p.

26. Lisingo, J., Dauby, J., Hardy, O., Boyemba, F., Makana, J.R., \& Ndjele, L. (2015). Structures spatiales de la richesse spécifique dans quelques blocs forestiers du nord-est du bassin congolais : implication pour la diversité régionale et la conservation. Geo-Eco-Trop, 39, 2 : 169-184.

27. Loris-Lukens (2009). Analyse de la diversité floristique dans les diverses strates des forêts denses de Masako (Kisangani, R.D.Congo). Mémoire de D.E.A, Université de Kisangani, Kisangani : 106 p.

28. Lubini, A.C. (1997). La végétation de la Réserve de la biosphère de Luki. Opera Bot. Belg. 10 : 1-155.

29. Marien, J.N. (2008). Forêt périurbaines et bois énergie : quels enjeux pour l'Afrique centrale. Rapport sur l'État des forêts d'Afrique centrale : 217-230.

30. Menga-Munkolo, P. (2012). Ecologie des peuplements naturels de Millettia laurentii De Wild. (Wenge) dans la région du lac MaïNdombe, en République Démocratique du Congo. Implication pour la gestion d'une espèce exploitée. Thèse UNIKIN, Kinshasa : 191 p.

31. Meunier, Q., Moumbogou, C., \& Doucet, J.L. (2015). Les arbres utiles du Gabon. Presses Agronomiques de Gembloux, 340 p.

32. Miabangana, E.S., Constantin Lubini-Ayingweu, C., \& Malaisse, F. (2016). Analyse floristique et phytogéographique de la forêt de la Djoumouna (République du Congo). Geo-Eco-Trop, 40-2, n.s.: 175190.

33. Miabangana, E.S. \& Hondjuila-Miokono, E.D. (2015). Contribution aux études ethnobotaniques et floristiques de la forêt de la Djoumouna, République du Congo : Les plantes comestibles. Afrique SCIENCE 11(4) : $227-240$. 
34. Miabangana, E.S. (2019). Analyse floristique, phytogéographique et phytosociologique de la végétation insulaire et riveraine du fleuve Congo dans le Plateau des Cataractes (République du Congo). Thèse de doctorat, Université de Kinshasa, Kinshasa : 303 p. + 91 annexes.

35. N’da, D.Hy., Adou, Y.C. Y., N’guessan, K.E., Koné, M., \& Sagne, Y.Ch. (2008). Analyse de la diversité floristique du parc national de la Marahoué, Centre-Ouest de la Côte d'Ivoire. Afrique Science 04 (03) : 552-579.

36. Ngomanda, A., Chepstow-Lusty, A., Makaya, M., Favier, C., Schevin, P., Maley, J.,Fontugne, M., Oslisly, R., \& Jolly, D. (2009). Western equatorial African forest-savanna mosaics: a legacy of late Holocene climatic change? Clim. Past, 5, 647-659.

37. Nshimba, S. M. H. (2008). Etude floristique, écologique et phytosociologique des forêts de l'île Mbiye à Kisangani (R.D. Congo), Thèse de doct ULB, Bruxelles : 272 p.

38. Nusbaumer, L., Gautier, L., Chatelain, C., \& Spichiger, R. (2005). Structure et composition floristique de la Forêt Classée du Scio (Côte d'Ivoire). Etude descriptive et comparative. Candollea 60 : 393-443.

39. Omatoko, J., Nshimba, H., Bogaert, J., Lejoly, J., Shutsha, R., Shaumba, J.P., Asimonyio, J., \& Ngbolua, K.N. (2015). Etudes floristique et structurale des peuplements sur sols argileux à Pericopsis elata et sableux à Julbernardia seretii dans la forêt de plaine d'UMA en République Démocratique du Congo. International Journal of Innovation and Applied Studies, Vol. 13 No. 2 Oct. pp. 452-463.

40. Pascal, J.P. (2003). Notions sur les structures et dynamique des forêts tropicales humides. Rev. For. LV. Numéro spécial, pp. 118-130.

41. Pavillard, J. (1955). Éléments de sociologie végétale. Paris. Actualités scientifiques, $102 \mathrm{p}$

42. Pierlot, R. (1966). Structure et composition de forêts denses d'Afrique centrale, Spécialement celles du Kivu. Ac. Roy. Se. XVI-4, Bruxelles, 367 p.

43. Pitman Nigel, C.A., John, W.T., Miles, R.S., Percy, N.V., David, A.N., Carlos, E.C., Walter, A.P., \& Milton, A. (2001). Dominance and distribution of tree species in upper Amazonian terra firme forest. Ecology, 82, 2101-2117.

44. Reitsma, J.M. (1988). Forest vegetation of Gabon (végétation forestière du Gabon).

45. Tropenbos Tech. Sér. 1 : 142 p. Wageningen.

46. Rollet, B. (1983). La régénération naturelle dans les trouées. Bois et Forêts des Tropiques 201, 3-34 ; 202, 19-33. 
47. Samba-Kimbata, M.J. (1978). Le climat Bas-Congolais. Dijon, Université de Dijon, thèse de 3ème cycle, Géographie 280 p., 132 fig. 64 table.

48. Sita, P. \& Moutsamboté, JM. (1988). Catalogue des plantes vasculaires du Congo. C.E.R.VE./ORSTOM, Brazzaville: 195 p.

49. Sonké, B. (1998). Etudes floristiques et structurales des forêts de la Réserve de faune du Dja (Cameroun). Th. Doct. En Sciences, ULB. $267 \mathrm{p}$.

50. Sörenson, T. (1948). A method of establishing groups of aqual amplitude in plant sociology based on similarity of species content and its application to analyses of the vegetation on Danish common. Kong. Danske videns. Selskob biolg. Sckr. Kjobenhavn, 4 : 1-34.

51. Tailfer, Y. (1990). La Forêt dense d'Afrique centrale - Identification pratique des principaux arbres. Agence de Coopération Culturelle et Technique et CTA, Wageningen, Tomes 1(456p) et 2 (1271p).

52. Tsoumou, B. R., Lumandé, K. J., Kampé, J. P., \& Nzila, J. D. (2016). Estimation de la quantité de Carbone séquestré par la Forêt Modèle de Dimonika (Sud-ouest de la République du Congo). Revue Scientifque et Technique Forêt et Environnement du Bassin du Congo Volume 6. P. 39 .

53. Vande Weghe, J.P., Bidault, E., \& Stévart, T. (2016). Plantes à fleurs du Gabon. Une introduction à la flore des angiospermes. (Col.Marc S.M. Sosef) Agence Nationale des Parcs Nationaux (ANPN), Libreville, Gabon : 792 p.

54. Vincens, A., Elenga, H., Schartz, D., de Nanur, C., Bertraux, J., Fournier, M., \& Dechamps, R. (2000). « Histoire des écosystèmes forestiers du Sud-Congo depuis 6000 ans ». In : Servant M., ServantVildary S. (eds) Dynamique à long terme des Ecosystèmesforestiers intertropicaux, 375-379. Mémoire Unesco, Paris

55. White, F. (1986). La végétation de l'Afrique. Mémoire accompagnant la carte de la végétation de l'Afrique. UNESCO/AETFA/UNSO, ORSTOM-UNESCO: 384 p.

56. White, L. \& Vande weghe, J.P. (2008). Patrimoine mondial Naturel d'Afrique centrale :Biens existants - Biens potentiels. Rapport de l'atelier de Brazzaville du 12-14 mars 2008.Unesco - CAWHFI, 98 p. 
ANNEXES : LISTE FLORISTIQUE ET DES PARAMETRES STRUCTURAUX

Légende : $\mathrm{St}_{\mathrm{rel}}$ : Surface terrière relatif ; $\mathrm{Do}_{\mathrm{rel}}$ : Dominance relative $; \mathrm{F}_{\mathrm{rel}}:$ Fréquence relative $; \mathrm{IVI}_{\mathrm{rel}}$ : Indice d'Importance relative

\begin{tabular}{|c|c|c|c|c|c|c|c|}
\hline $\mathrm{N}^{\circ}$ & Espèces de $\mathrm{D}_{1,3} \geq 10 \mathrm{~cm}$ du peuplement forestier & Famille/Sous famille & Densité & Strel & Dorel & Frel $(\%)$ & IVI rel \\
\hline 1 & Albizia ferruginea (Guill. et Perr.) Benth. & Fabaceae-Mimosoideae & 3 & 1,887 & 0,068 & 0,32051 & 0,677 \\
\hline 2 & Albizia gummifera (J.F. Gmel.) C.A. Sm. & Fabaceae-Mimosoideae & 6 & 2,63 & 0,095 & 0,32051 & 0,993 \\
\hline 3 & Alchornea cordifolia (Schumach. et Thonn.) Müll.Arg. & Euphorbiaceae & 1 & 0,008 & 3E-04 & 0,16026 & 0,257 \\
\hline 4 & Alchornea floribunda Müll.Arg. & Euphorbiaceae & 8 & 0,528 & 0,019 & 1,12179 & 1,911 \\
\hline 5 & Allanblackia floribunda Oliv. & Clusiaceae & 25 & 29,13 & 1,048 & 0,80128 & 4,256 \\
\hline 6 & Amphimas ferrugineus Pierre ex Pellegr. & Fabaceae-Faboideae & 1 & 0,042 & 0,001 & 0,32051 & 0,418 \\
\hline 7 & Anisophyllea meniaudi Aubrév. et Pellegr. & Anisphylleaceae & 1 & 0,011 & 4E-04 & 0,32051 & 0,417 \\
\hline 8 & Annonaceae ind ( $\left.\mathrm{N}^{\circ}: 1113\right)$ & Annonaceae & 2 & 0,126 & 0,005 & 0,16026 & 0,357 \\
\hline 9 & Anonidium mannii (Oliv.) Engl. et Diels & Annonaceae & 3 & 1,227 & 0,044 & 0,32051 & 0,653 \\
\hline 10 & Anopyxis klaineana (Pierre) Engl & Rhizophoraceae & 1 & 0,332 & 0,012 & 0,16026 & 0,268 \\
\hline 11 & Anthocleista schweinfurthii Gilg & Gentianaceae & 2 & 0,385 & 0,014 & 0,32051 & 0,527 \\
\hline 12 & Anthonotha gilletii (De Wild.) J.Léonard & Fabaceae-Caesalpinioideae & 2 & 0,332 & 0,012 & 0,16026 & 0,365 \\
\hline 13 & Anthonotha macrophylla P.Beauv. & Fabaceae-Caesalpinioideae & 9 & 4,988 & 0,179 & 0,64103 & 1,687 \\
\hline 14 & Aoranthe cladantha (K.Schum.) Somers & Rubiaceae & 6 & 0,899 & 0,032 & 0,64103 & 1,251 \\
\hline 15 & Aphanocalyx microphyllus (Harms) Wieringa subsp. microphyllus & Fabaceae-Caesalpinioideae & 4 & 2,27 & 0,082 & 1,60256 & 2,069 \\
\hline 16 & Aptandra zenkeri Engl. & Aptandraceae & 1 & 0,126 & 0,005 & 0,16026 & 0,261 \\
\hline 17 & Barteria dewevrei De Wild. et T. Durand & Passifloraceae & 1 & 0,018 & $6 \mathrm{E}-04$ & 0,16026 & 0,257 \\
\hline 18 & Beilschmiedia minutiflora (Meisn.) Benth. et Hook. f. & Lauraceae & 1 & 0,008 & 3E-04 & 1,12179 & 1,218 \\
\hline 19 & Berlinia auriculata Benth. & Fabaceae-Caesalpinioideae & 1 & 0,049 & 0,002 & 0,16026 & 0,258 \\
\hline 20 & Berlinia grandiflora (Vahl) Hutch. et Dalziel & Fabaceae-Caesalpinioideae & 3 & 0,255 & 0,009 & 0,32051 & 0,618 \\
\hline 21 & Blighia welwitschii (Hiern) Radlk. & Sapindaceae & 4 & 1,72 & 0,062 & 0,48077 & 0,928 \\
\hline 22 & Bosqueiopsis gilletii De Wild. et T.Durand & Moraceae & 15 & 13,07 & 0,47 & 1,60256 & 3,517 \\
\hline 23 & Canarium schweinfurthii Engl. & Burseraceae & 3 & 0,156 & 0,006 & 0,48077 & 0,775 \\
\hline 24 & Carapa procera DC. & Meliaceae & 8 & 0,156 & 0,006 & 1,44231 & 2,218 \\
\hline 25 & Cathormion altissimum (Hook.f.) Hutch. et Dandy & Fabaceae-Mimosoideae & 1 & 0,031 & 0,001 & 0,16026 & 0,258 \\
\hline 26 & Ceiba pentandra (L.) Gaertn. & Malvaceae-Bombacoideae & 2 & 0,442 & 0,016 & 0,32051 & 0,529 \\
\hline 27 & Celtis adolfi-friderici Engl. & Cannabaceae & 1 & 0,071 & 0,003 & 0,32051 & 0,419 \\
\hline 28 & Celtis tessmannii Rendle & Cannabaceae & 10 & 8,501 & 0,306 & 2,08333 & 3,352 \\
\hline 29 & Chaetocarpus africanus Pax & Euphorbiaceae & 14 & 4,449 & 0,16 & 1,76282 & 3,27 \\
\hline 30 & Chlamydocola chlamydantha (K.Schum.) M.Bodard & Malvaceae-Sterculioideae & 1 & 0,008 & $3 \mathrm{E}-04$ & 1,12179 & 1,218 \\
\hline 31 & Chrysophyllum subnudum Baker & Sapotaceae & 16 & 22,15 & 0,797 & 2,88462 & 5,221 \\
\hline 32 & Cleistanthus caudatus $\mathrm{Pax}$ & Phyllanthaceae & 2 & 0,113 & 0,004 & 0,16026 & 0,357 \\
\hline
\end{tabular}




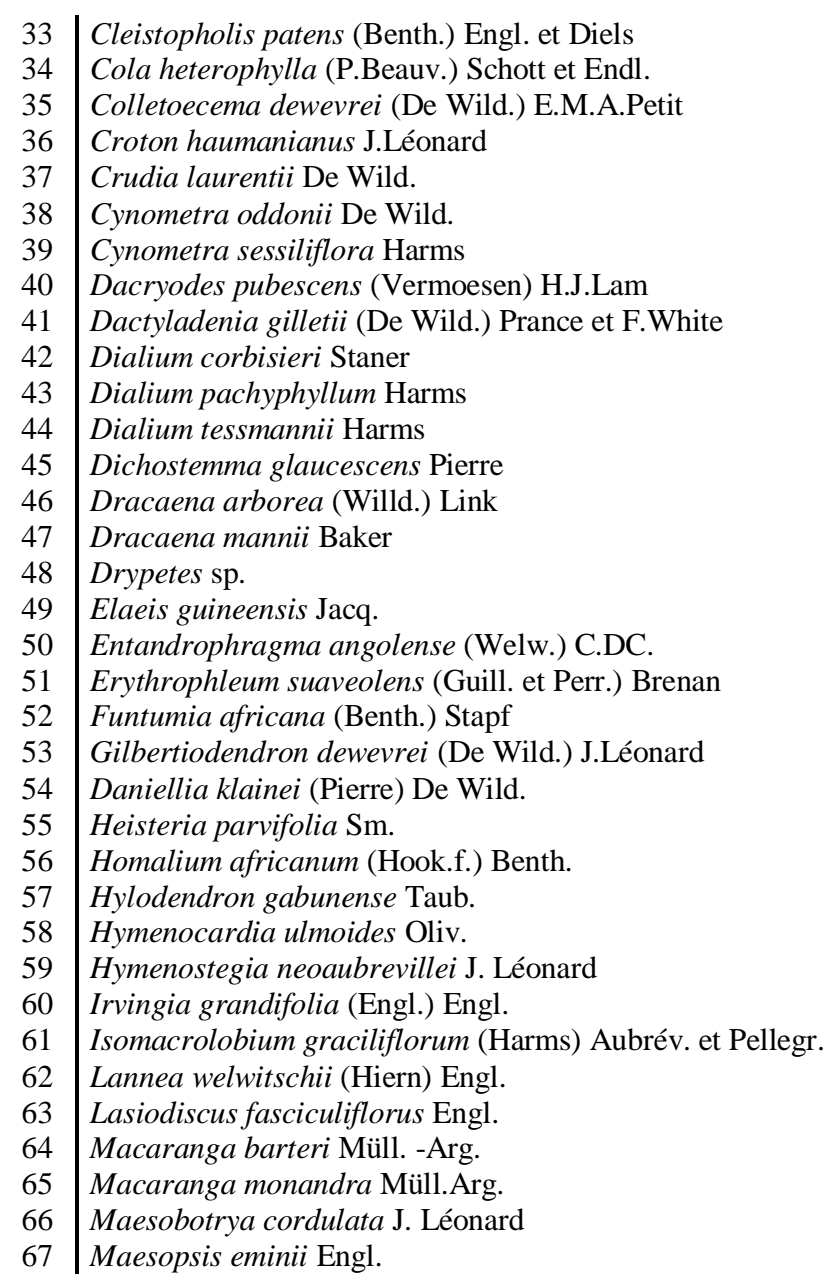

\begin{tabular}{|l} 
Annonaceae \\
Malvaceae-Sterculioideae \\
Rubiaceae \\
Euphorbiaceae \\
Fabaceae-Caesalpinioideae \\
Fabaceae-Caesalpinioideae \\
Fabaceae-Caesalpinioideae \\
Burseraceae \\
Chrysobalanaceae \\
Fabaceae-Caesalpinioideae \\
Fabaceae-Caesalpinioideae \\
Fabaceae-Caesalpinioideae \\
Euphorbiaceae \\
Asparagaceae \\
Asparagaceae \\
Putranjivaceae \\
Arecaceae \\
Meliaceae \\
Fabaceae-Caesalpinioideae \\
Apocynaceae \\
Fabaceae-Caesalpinioideae \\
Fabaceae-Caesalpinioideae \\
Olacaceae \\
Salicaceae \\
Fabaceae-Caesalpinioideae \\
Phyllanthaceae \\
Fabaceae-Caesalpinioideae \\
Irvingiaceae \\
Fabaceae-Caesalpinioideae \\
Anacardiaceae \\
Rhamnaceae \\
Euphorbiaceae \\
Euphorbiaceae \\
Phyllanthaceae \\
Rhamnaceae \\
\end{tabular}

\begin{tabular}{|l|c|r|c|}
0,049 & 0,002 & 0,16026 & 0,258 \\
0,152 & 0,005 & 2,5641 & 2,955 \\
0,085 & 0,003 & 0,80128 & 0,997 \\
0,196 & 0,007 & 0,32051 & 0,52 \\
1,13 & 0,041 & 0,16026 & 0,297 \\
0,196 & 0,007 & 0,32051 & 0,52 \\
2,011 & 0,072 & 0,80128 & 1,355 \\
1,584 & 0,057 & 0,64103 & 0,987 \\
1,207 & 0,043 & 0,48077 & 0,909 \\
1,767 & 0,064 & 0,48077 & 0,929 \\
0,152 & 0,005 & 0,80128 & 1,095 \\
1,767 & 0,064 & 0,32051 & 0,673 \\
44,06 & 1,586 & 2,08333 & 6,941 \\
0,283 & 0,01 & 0,16026 & 0,267 \\
24,19 & 0,871 & 0,96154 & 3,95 \\
0,008 & $3 \mathrm{E}-04$ & 0,32051 & 0,417 \\
105,3 & 3,79 & 2,40385 & 9,466 \\
0,08 & 0,003 & 0,16026 & 0,259 \\
0,212 & 0,008 & 1,60256 & 1,995 \\
4,264 & 0,153 & 0,64103 & 1,468 \\
57,15 & 2,056 & 1,92308 & 6,289 \\
0,332 & 0,012 & 0,16026 & 0,268 \\
21,24 & 0,764 & 2,40385 & 5,767 \\
0,011 & $4 \mathrm{E}-04$ & 0,16026 & 0,257 \\
0,175 & 0,006 & 0,16026 & 0,359 \\
67,64 & 2,434 & 3,04487 & 8,847 \\
7,548 & 0,272 & 0,80128 & 1,747 \\
1,72 & 0,062 & 0,32051 & 0,575 \\
0,95 & 0,034 & 0,32051 & 0,932 \\
1,327 & 0,048 & 0,48077 & 0,914 \\
0,008 & $3 \mathrm{E}-04$ & 0,16026 & 0,257 \\
0,071 & 0,003 & 0,32051 & 0,419 \\
19,09 & 0,687 & 1,28205 & 3,99 \\
1,791 & 0,064 & 0,96154 & 2,181 \\
1,651 & 0,059 & 0,64103 & 1,278
\end{tabular}




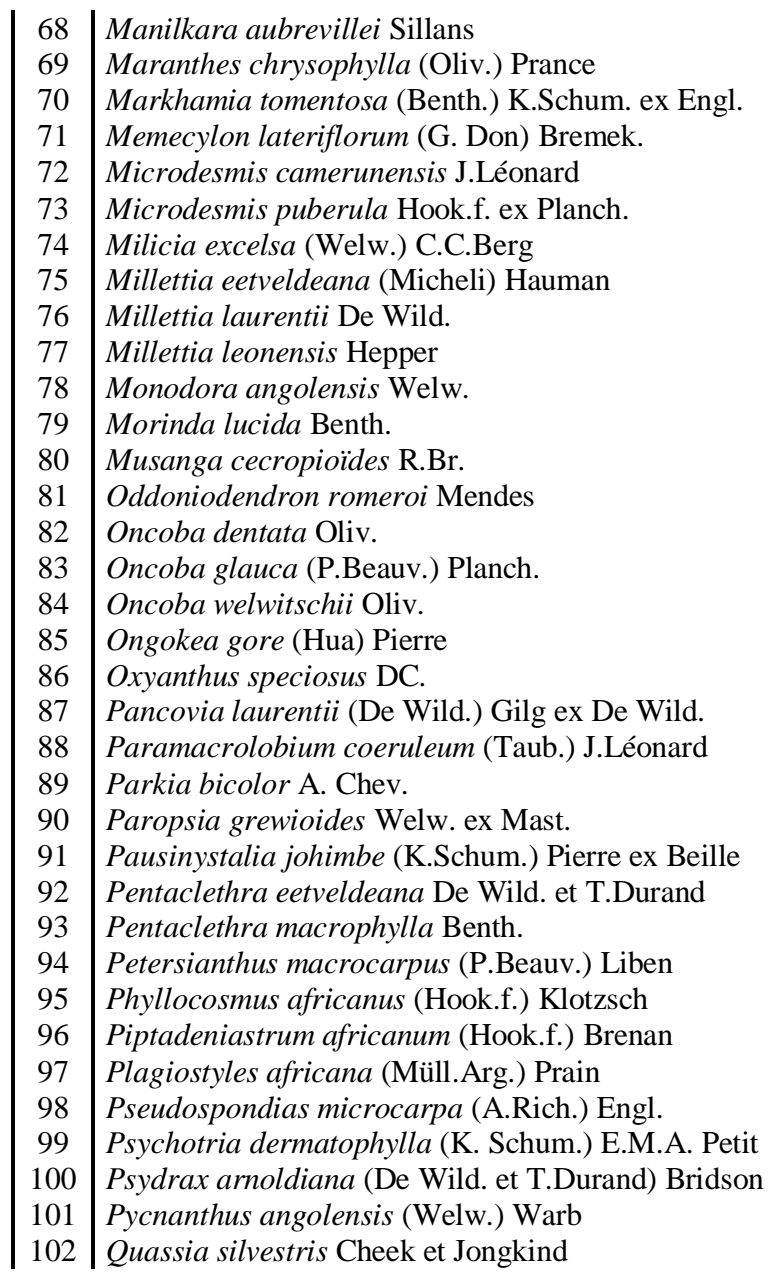

\begin{tabular}{|l} 
Sapotaceae \\
Chrysobalanaceae \\
Bignoniaceae \\
Melastomataceae \\
Pandaceae \\
Pandaceae \\
Moraceae \\
Fabaceae-Faboideae \\
Fabaceae-Faboideae \\
Fabaceae-Faboideae \\
Annonaceae \\
Rubiaceae \\
Urticaceae \\
Fabaceae-Caesalpinioideae \\
Salicaceae \\
Salicaceae \\
Salicaceae \\
Aptandraceae \\
Rubiaceae \\
Sapindaceae \\
Fabaceae-Caesalpinioideae \\
Fabaceae-Mimosoideae \\
Passifloraceae \\
Rubiaceae \\
Fabaceae-Mimosoideae \\
Fabaceae-Mimosoideae \\
Lecythidaceae \\
Ixonanthaceae \\
Fabaceae-Mimosoideae \\
Euphorbiaceae \\
Anacardiaceae \\
Rubiaceae \\
Rubiaceae \\
Myristicaceae \\
Simaroubaceae \\
\end{tabular}

\begin{tabular}{|c|c|c|c|}
21,16 & 0,761 & 1,76282 & 4,353 \\
0,138 & 0,005 & 0,16026 & 0,454 \\
0,071 & 0,003 & 0,32051 & 0,419 \\
0,011 & $4 \mathrm{E}-04$ & 0,16026 & 0,257 \\
0,283 & 0,01 & 0,64103 & 1,229 \\
0,283 & 0,01 & 1,44231 & 2,03 \\
1,093 & 0,039 & 0,16026 & 0,488 \\
0,161 & 0,006 & 0,48077 & 0,872 \\
15,62 & 0,562 & 0,96154 & 2,775 \\
0,554 & 0,02 & 0,48077 & 0,982 \\
0,554 & 0,02 & 0,32051 & 0,822 \\
0,011 & $4 \mathrm{E}-04$ & 0,16026 & 0,257 \\
86,26 & 3,104 & 1,12179 & 6,536 \\
0,636 & 0,023 & 0,96154 & 1,369 \\
0,096 & 0,003 & 1,44231 & 1,638 \\
0,018 & $6 \mathrm{E}-04$ & 0,16026 & 0,257 \\
3,267 & 0,118 & 1,28205 & 2,266 \\
0,062 & 0,002 & 0,16026 & 0,259 \\
1,629 & 0,059 & 1,12179 & 2,432 \\
0,246 & 0,009 & 0,96154 & 1,259 \\
19,48 & 0,701 & 0,96154 & 2,625 \\
0,708 & 0,025 & 0,32051 & 0,538 \\
21,73 & 0,782 & 2,08333 & 5,368 \\
0,865 & 0,031 & 0,64103 & 1,057 \\
1120 & 40,32 & 3,52564 & 54,05 \\
584,5 & 21,03 & 3,20513 & 30,59 \\
10,29 & 0,37 & 0,80128 & 2,134 \\
5,599 & 0,201 & 0,64103 & 1,42 \\
0,785 & 0,028 & 0,32051 & 0,541 \\
122,7 & 4,416 & 3,04487 & 12,08 \\
23,41 & 0,843 & 0,80128 & 2,991 \\
5,683 & 0,205 & 0,96154 & 2,225 \\
96,94 & 3,489 & 1,92308 & 8,492 \\
10,46 & 0,377 & 1,28205 & 2,717 \\
113,5 & 4,083 & 1,76282 & 8,637
\end{tabular}




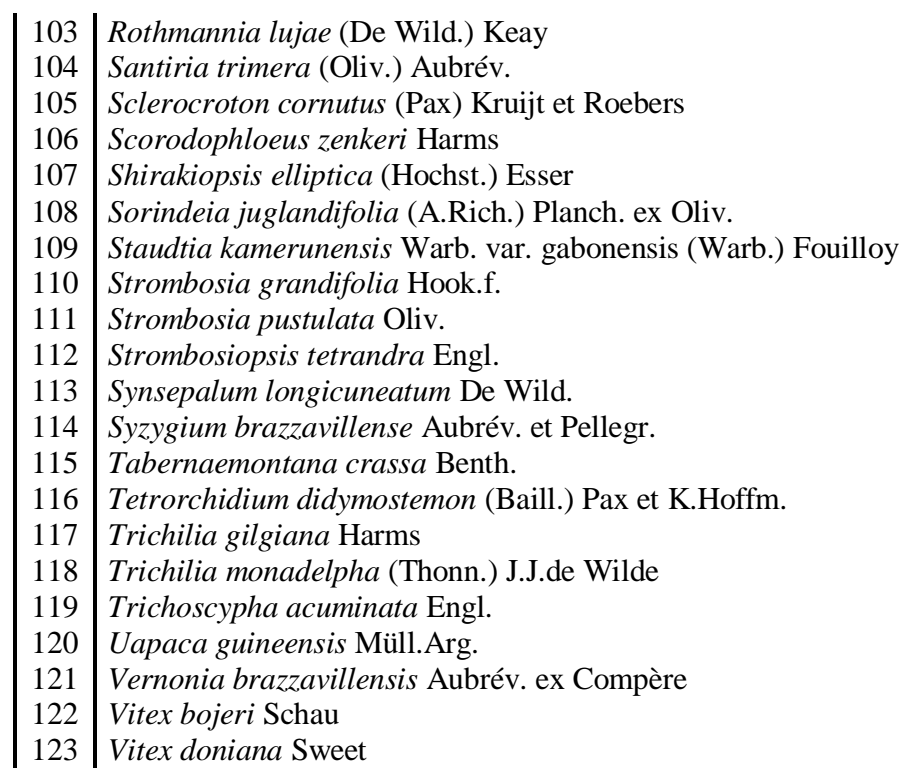

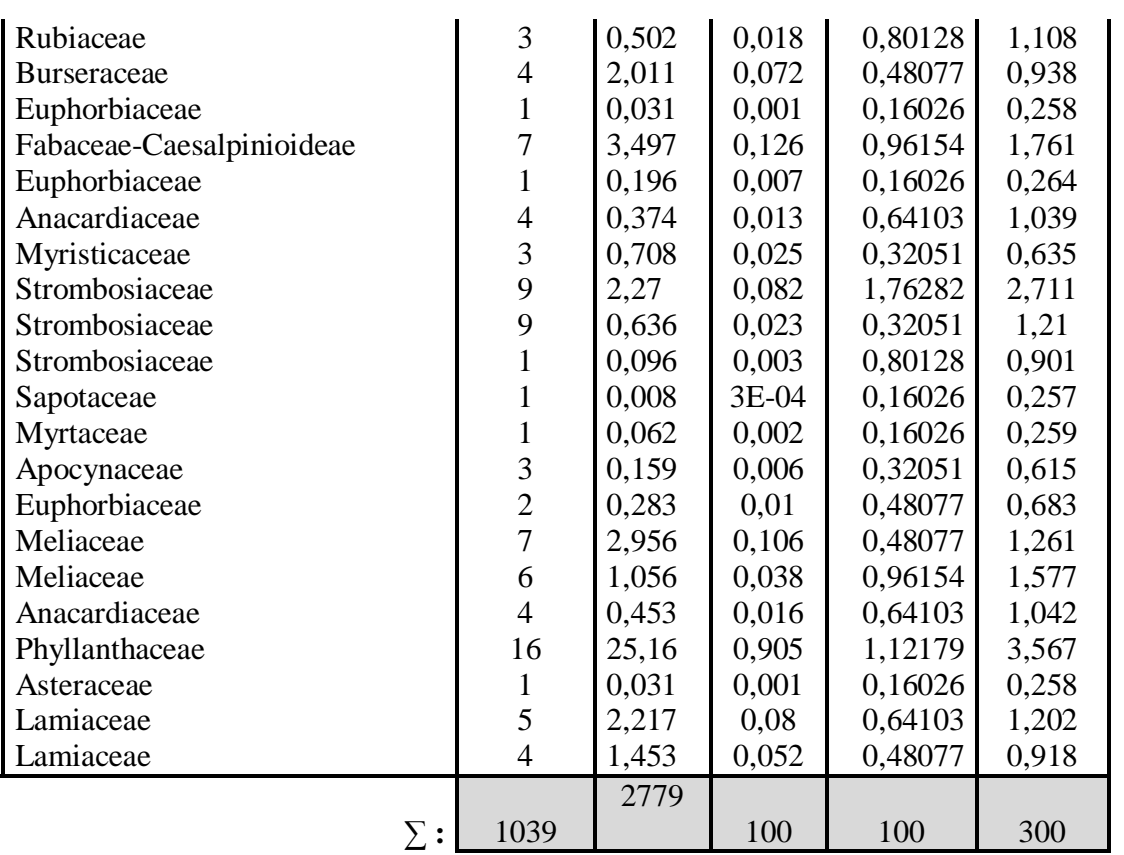

